\title{
Smart dendrimer-based nanogel for enhancing 5-fluorouracil loading efficiency against MCF7 cancer cell growth
}

\author{
PHUNG NGAN LE ${ }^{1,3}$, NGOC HOA NGUYEN ${ }^{2}$, CUU KHOA NGUYEN ${ }^{3, *}$ and \\ NGOC QUYEN TRAN ${ }^{1,3}$ \\ ${ }^{1}$ Institute of Research and Development, Duy Tan University, Da Nang City 550000, Vietnam \\ ${ }^{2}$ Department of Food and Chemical Engineering, University of Food Industry, HCMC 70000, Vietnam \\ ${ }^{3}$ Institute of Applied Materials Science, Vietnam Academy Science and Technology, Ho Chi Minh City 700000, Vietnam
}

MS received 30 November 2015; accepted 24 February 2016

\begin{abstract}
Nano-carriers are not only evaluated as a novel kind of drug delivery, but also expected to bypass the critical bottleneck of conventional cancer chemotherapeutics. Among them, thermo-sensitive nanogel draws much attention due to its efficacy in the loading and release of hydrophobic drugs. In the study, we developed a promising thermosensitive polymer-grafted dendrimer to enhance drug-loading efficiency, which was prepared from conjugation of thermo-sensitive carboxylic-terminated poly(N-isopropylacrylamide) polymer (PNIPAM) with polyamidoamine (PAMAM) dendrimer (G3.0). The obtained copolymer structure and molecular weight were confirmed by proton nuclear magnetic resonance $\left({ }^{1} \mathrm{H}\right.$ NMR) and gel permeation chromatography (GPC), respectively. Morphology of the nanocarrier was observed around 120-150 $\mathrm{nm}$ by transmission electron microscopy (TEM) and $200 \mathrm{~nm}$ by dynamic light scattering (DLS). The nanocarrier exhibited the higher drug loading (DL = 7.79\%) and entrapment efficiency $(\mathrm{EE}=\mathbf{4 2 . 2 5 \%}$ ) of 5-FU compared to PAMAM dendrimer G3.0 (DL $=\mathbf{2 . 2 5 \%}$ and EE $=\mathbf{1 1 . 5 2 \%})$. In-vitro test, the 5-FU-loaded in PAMAM G3.0-PNIPAM could release approximately $40 \%$ of the encapsulated drug at pH $=7.4$ after 5 days tracking, while the cumulative anticancer drugs achieved nearly two-fold increase (around $75 \%$ ) at pH 5.5 during the same time. Moreover, the cytotoxicity assay results also indicated that the drug-loaded nanocarrier exhibited a significant growth inhibition of the MCF-7 cancer cell. The obtained resulted possibly offered a great potential of the nanocarrier which may be utilized in delivering other anticancer drugs or dual drugs for chemotherapy in future.
\end{abstract}

Keywords. Thermo-sensitive; smart dendrimer; nanocarriers; MCF-7 cancer cells.

\section{Introduction}

Chemotherapy drugs, typically 5-FU, cisplatin, palitaxcel (taxol) and doxorubicin have come a long way in the oncology regiment for several years. However, several side effects of the drugs such as nausea, vomiting, nephrotoxicity, electrolyte disturbances and drug resistance greatly impact cancer patients $[1,2]$. Since the end of the 20th century, nanoscale carriers have attracted great interest on efforts to enhance drug distribution in the body and achieve effective cancer treatments with reduced toxic side effects. In other words, various types of the nanodrug delivery such as liposomes, dendrimers, solid-lipid nanoparticles and virus-like nanoparticles as well as a wide branch of the polymeric nanocarrier, have been potential candidates for the alternative therapies in the pharmacological treatment of various diseases $[3,4]$.

Following the trend, polyamidoamine (PAMAM) dendrimer is also one of the most studied nanocarriers for drugdelivery system owning to the advantage of its well-defined nanosize. The structure can be easily modified to change the

*Author for correspondence (nckhoavnn@yahoo.com) chemical properties of the system as well as drugs loaded in their highly inner cavities or encapsulated/functionalized via (non)-covalent interactions to enhance drugs solubility and control delivery [5-7]. However, there are few disadvantages accompanied with PAMAM dendrimer in drug-delivery system including haemolytic toxicity and cell lysis, which happen due to strong interactions of the positively-charged dendrimer and the negatively-charged cell membrane resulting in membrane disruption [8,9]. Several effective strategies have been developed to modify PAMAM dendrimer to minimize its cytotoxicity $[10,11]$. These modifications showed the significant reduction in haemolytic and cytotoxic activities as well as in the improvement of dendrimer biocompatibility.

Similar to the dendrimer-based nanocarriers, thermo-sensitive polymers are emerging materials to manipulate nanogels or nanoparticles for delivering anticancer drugs. Thermo-sensitive copolymers-based nanogels such as poly(N-(2-hydroxypropyl) methacrylamide (HPMA) (completed clinical phase II) and poly(N-isopropylacrylamide) (PNIPAM) have also been developed to deliver the drugs $[12,13]$. The polymer solution can be responsive to temperature changes of the external environment to form nanogel above $32^{\circ} \mathrm{C}$, a very 
useful value for biomedical applications, since it undergoes a reversible phase transition as a result of the coil-to-globule transition that could result in enhancing the hydrophobic drug encapsulation via hydrophobic interaction [14-19].

In this study, we introduce a smart PNIPAM-conjugated PAMAM dendrimer nanocarrier which utilizes the inner cavity space of dendrimer molecule and hydrophobic interaction of PNIPAM resulting in increasing drug-loading capacity and controlling its delivery (as demonstrated in figure 1). Moreover, the drug nanocarriers may be expected to increase the residence time of the drug in blood circulation by its stealth properties in the blood plasma.

\section{Materials and methods}

\subsection{Materials}

Carboxylic acid terminated poly(N-isopropylacrylamide) (PNIPAM-COOH; Mw = 7000) was purchased from SigmaAldrich. 5-Fluorouracil (5-FU) was purchased from Merck Chemicals. PAMAM G3.0 dendrimer (G3.0; Mw = 6900) was prepared in Department of Materials and Pharmacy Chemistry (Institute of Applied Materials Science) following the procedure reported by Tomalia et al [4,20]. 1-(3-Dimethylaminopropyl)-3-ethylcarbodimide hydrochloride (EDC) and

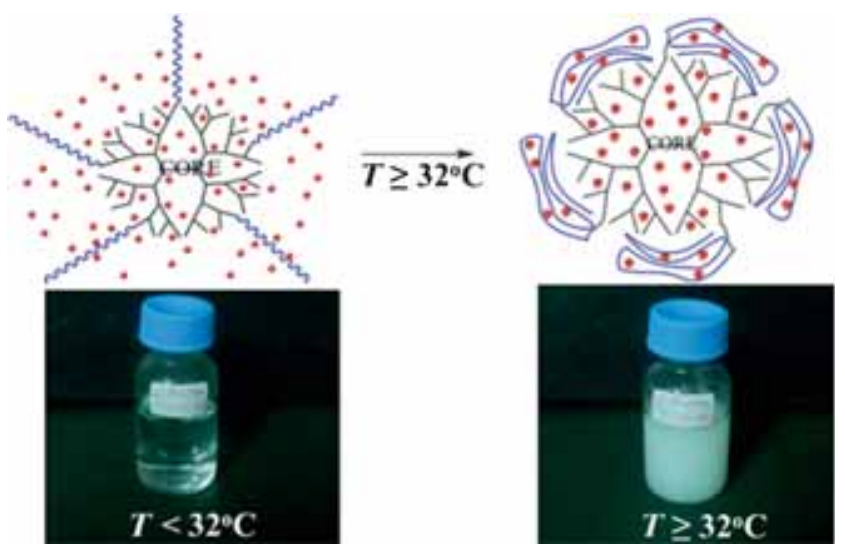

Figure 1. Mechanism for enhancing drug-loading capacity of G3.0-PNIPAM nanocarrier.
N-hydroxy succinimide (NHS) were purchased from Acros Organics. Regenerated cellulose MWCO 3500-5000 Da and 12000-14000 Da dialysis bags were from Spectrum Laboratories Inc. All other chemicals and solvent were used without further purification.

\subsection{Synthesis of PAMAM G3-PNIPAM}

A solution of PAMAM G3.0 (315 mg; $0.045 \mathrm{mmol}$ ) and distilled water $(3 \mathrm{ml})$ were added into the two-neck flask. Then, $\mathrm{HCl}$ solution (10\%) was added drop-wise into the cold solution until the $\mathrm{pH}$ value is around 6.5. Twelve millilitre of PNIPAM-COOH solution (1.75 g; $0.25 \mathrm{mmol}$ ) was added into the mixture under a constant stirring condition below $30^{\circ} \mathrm{C}$. And then, NHS $(0.5 \mathrm{mmol})$ and $\operatorname{EDC}(0.5 \mathrm{mmol})$ were added into the solution to activate PNIPAM-COOH. The reaction was kept at a constant temperature below $30^{\circ} \mathrm{C}$ for $24 \mathrm{~h}$. Then, the solution was dialyzed by regenerated cellulose MWCO 12000-14000 Da dialysis bags in methanol for 4 days. $1.95 \mathrm{~g}$ of PAMAM G3.0-PNIPAM was obtained from the dialyzed sample via rotary evaporation (figure 2). The copolymer was characterized by proton nuclear magnetic resonance $\left({ }^{1} \mathrm{H} \mathrm{NMR}\right)$ and gel permeation chromatography (GPC) measurements.

\subsection{5-FU loading in PAMAM G3-PNIPAM}

First, PAMAM G3.0-PNIPAM was dissolved in distilled water $\left(100 \mathrm{mg} 1.5 \mathrm{ml}^{-1}\right)$. Then, $20 \mathrm{mg}$ of 5 -FU was added steadily into the copolymer solution and then magnetically stirred for $24 \mathrm{~h}$ for drug-loading. The solution was dialyzed in distilled water by dialysis membrane in the range of $\mathrm{MWCO} 3500-5000 \mathrm{Da}$ at $37^{\circ} \mathrm{C}$ and triplicate to remove completely free 5-FU. Then, the obtained 5FU-loaded PAMAM G3.0-PNIPAM sample was lyophilized to be used for further studies. Unloaded drug was determined by the withdrawn solution from dialysis measured high-performance liquid chromatography (HPLC). This result indicated the amount of 5-FU drug loaded in the nanocarrier. The drug loading (DL\%) and entrapment
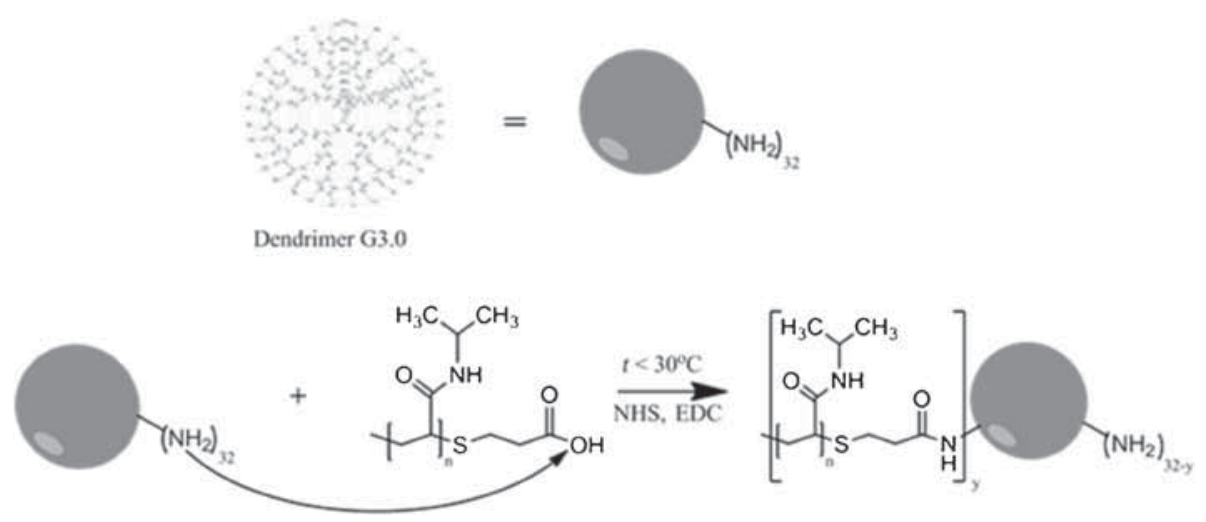

Figure 2. Synthetic process of thermosensitive nanocarriers (G3.0-PNIPAM). 
efficiency (EE\%) of 5-FU in thermo-sensitive dendrimer were calculated from the following equations.

$\mathrm{DL}(\%)=\frac{\text { Mass of loaded 5-FU }}{\text { (Mass of loaded 5-FU }+ \text { mass of polymer) }} \times 100 \%$,

$\mathrm{EE}(\%)=\frac{(\text { Total } 5-\mathrm{FU} \text { amount }- \text { free } 5-\mathrm{FU} \text { amount })}{(\text { Initial mass of } 5-\mathrm{FU})} \times 100 \%$.

\subsection{In-vitro drug release}

The 5-FU loaded PAMAM G3.0-PNIPAM or free 5-FU was dissolved in deionized water. Then, the solutions were added to the dialysis bag $3500 \mathrm{Da}$ and dialyzed with 15 $\mathrm{ml}$ phosphate buffer saline (PBS) solutions at $\mathrm{pH} 7.4$ and 5.5 in two separate tubes at $37^{\circ} \mathrm{C}$. At the predetermined time interval, $1 \mathrm{ml}$ of dialyzed solution was drawn to determine 5-FU release and replaced with an equivalent volume of PBS into each tube. 5-FU release was determined by the HPLC method with the absorption wavelength at $260 \mathrm{~nm}$ and mobile phase by acetonitrile and $\mathrm{H}_{2} \mathrm{O}$ in the ratio of $1: 9 \mathrm{v} / \mathrm{v}$.

\subsection{Cytotoxicity assays}

The experiment was conducted via sulfordodamine B (SRB) colorimetric assay at Faculty of Biology, University of Science, Vietnam National University in Ho Chi Minh City. In other words, PAMAM G3.0, PAMAM G3.0-PNIPAM, free 5-FU and 5-FU loaded PAMAM G3.0-PNIPAM-5FU were optimized at the screening to test the inhibition capability of cell growth. The MCF-7 cell line preserved in liquid nitrogen was thawed to culture them to 4th generation. Then, MCF-7 cells were seeded in 96 well plates $\left(10^{4}\right.$ cells well $\left.{ }^{-1}\right)$ and allowed for $24 \mathrm{~h}$ for cell growth in culture medium with $5 \% \mathrm{CO}_{2}$ atmosphere at $37^{\circ} \mathrm{C}$. Then, the medium was replaced by the tested samples with a predetermined concentration and incubated for $48 \mathrm{~h}$ later. The culture medium was divided into 2 separate mediums, of which one considered as 'negative control', while another was 'blank sample' containing samples but without cells. After being incubated, the cells were fixed with $50 \%$ (wt./vol.) trichloroacetic acid and stained with $0.2 \%$ (wt./vol.) SRB for $20 \mathrm{~min}$. The samples were washed repeatedly (five times) and clearly with $1 \%(\mathrm{v} / \mathrm{v})$ acetic acid. Finally, the protein-bound dye was dissolved in $10 \mathrm{mM}$ Tris-baz solution to determine the optical density (OD) with the absorption wavelength at 492 and $620 \mathrm{~nm}$. Based on the OD results of control, blank and sample, the growth inhibition values were calculated [21,22].

\subsection{Characterizations}

Nuclear magnetic resonance (NMR) spectrum of copolymer (Bruker Advance 500 NMR spectrometer) was measured in methanol at $500 \mathrm{MHz}$. For GPC measurement, the test was performed on an Agilent model 1260 GPC. PAMAM G3.0PNIPAM was dissolved and measured in distilled tetrahydrofuran (THF). The solvent was also used as an eluent at a flow rate of $0.3 \mathrm{ml} \mathrm{min}^{-1}$. Transmission electron microscopy (TEM) images were obtained using a JEOL JEM-1010 operating at $100 \mathrm{kV}$, equipped with an AMT XR40 digital camera with $2 \mathrm{~K} \times 2 \mathrm{~K}$ pixels. Dendrimer samples were dissolved in water at $25^{\circ} \mathrm{C}$, dropped onto a carbon-coated $\mathrm{Cu}$ grid (EMSciences, Gibbstown, NJ) and dried at $37^{\circ} \mathrm{C}$ to observe morphology of the PAMAM G3.0-PNIPAM copolymer. Size distribution of the PAMAM G3.0-PNIPAM nanocarrier was recorded at $37^{\circ} \mathrm{C}$ with $\mathrm{SZ}-100$ nanoparticle analyser using the HORIBA patented carbon electrode cell (Horiba Instruments, Singapore Pte Ltd, Singapore).

\section{Results and discussions}

\subsection{Characterization of PAMAM G3.0 dendrimer and PAMAM G3.0-PNIPAM}

Spectra of PAMAM generation G3.0 (figure 3) showed that performed resonance signals of protons corresponding to typical protons in the dendrimer structure such as $-\mathrm{CH}_{2} \mathrm{CH}_{2} \mathrm{~N}<$ (a, $\delta$ 2.61-2.62 ppm), $-\mathrm{CH}_{2} \mathrm{CH}_{2} \mathrm{CO}-$ (b, $\delta 2.80-2.83 \mathrm{ppm}$ ), $-\mathrm{CH}_{2} \mathrm{CH}_{2} \mathrm{CONH}-(\mathrm{c}, \delta 2.38-2.40 \mathrm{ppm}),-\mathrm{CH}_{2} \mathrm{CH}_{2} \mathrm{NH}_{2}$

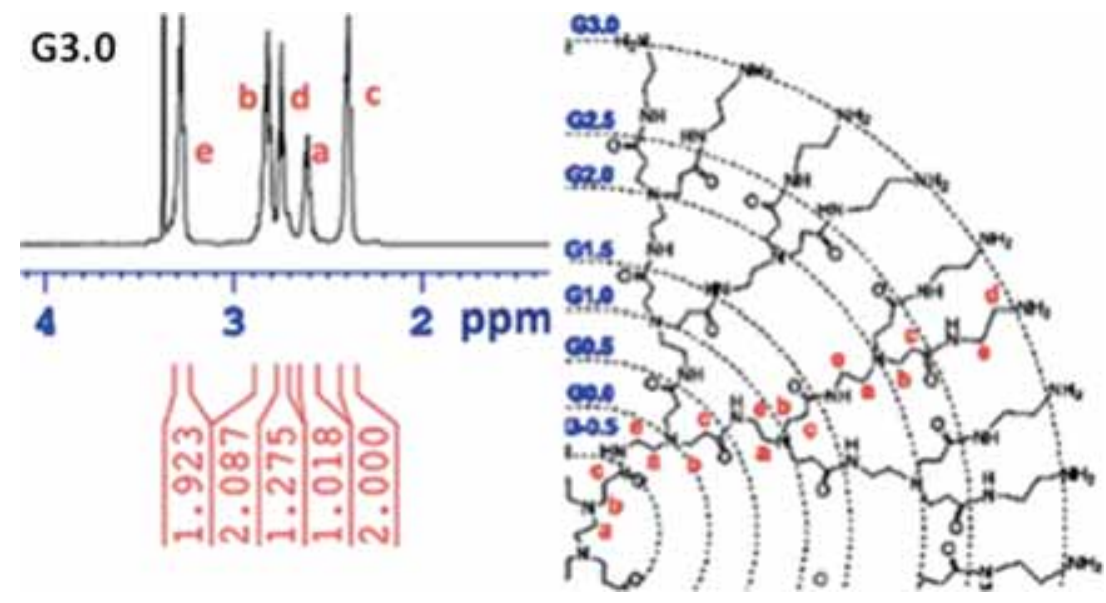

Figure 3. ${ }^{1} \mathrm{H}-\mathrm{NMR}$ spectrum of the PAMAM G3.0 dendrimer. 
(d, $\delta$ 2.74-2.76 ppm) and $-\mathrm{CONHCH}_{2} \mathrm{CH}_{2} \mathrm{~N}-(\mathrm{e}, \delta 3.26-$ $3.33 \mathrm{ppm}$ ). With large numbers of amine-terminating groups, PAMAM dendrimer could be easily functionalized with some functional groups such as carboxyl end group (HOOCPNIPAM). Besides some peaks in the dendrimer structure, typical proton signals of PAMAM G3.0-PNIPAM (figure 4) such as $\mathrm{CH}_{3}-$ (f), $-\mathrm{CH}_{2} \mathrm{CH}_{2} \mathrm{CONH}-(\mathrm{c}),-\mathrm{CH}_{2}-$ (h), $\mathrm{CH}\left(\mathrm{CH}_{3}\right)_{2} \mathrm{NHCO}-(\mathrm{g})$ and $-\mathrm{NHCOCH}\left(\mathrm{CH}_{2}\right)_{2}$ (i), notably, chemical shifts were reported in the range of $0.906-1.180$ (f). ${ }^{1} \mathrm{H}-\mathrm{NMR}$ spectra indicated that the area of peak (c) is in the range of 2.000-2.526. In other words, there was a high increase in the amide bond ( $\mathrm{CO}-\mathrm{NH})$ between carboxylic acid group (-COOH) of carboxylic acid-terminated PNIPAM and amine $\left(-\mathrm{NH}_{2}\right)$ group of PAMAM G3.0 dendrimer.

The molecular weight of the conjugated PAMAM and conversion degree of their conjugated derivatives were identified by the theoretical number of protons at specific positions in the dendrimers and the real number of the integral values of these protons appearance in ${ }^{1} \mathrm{H}$ NMR spectra. Based on the fed amount of moles $\mathrm{OC}-\mathrm{COOH}(x \%)$, numbers of PNIPAM-conjugated groups and molecular weight (Mw) of thermo-sensitive dendritic derivatives (table 1), formula 1 was used to calculate the conjugation efficacy (table 1) [20].

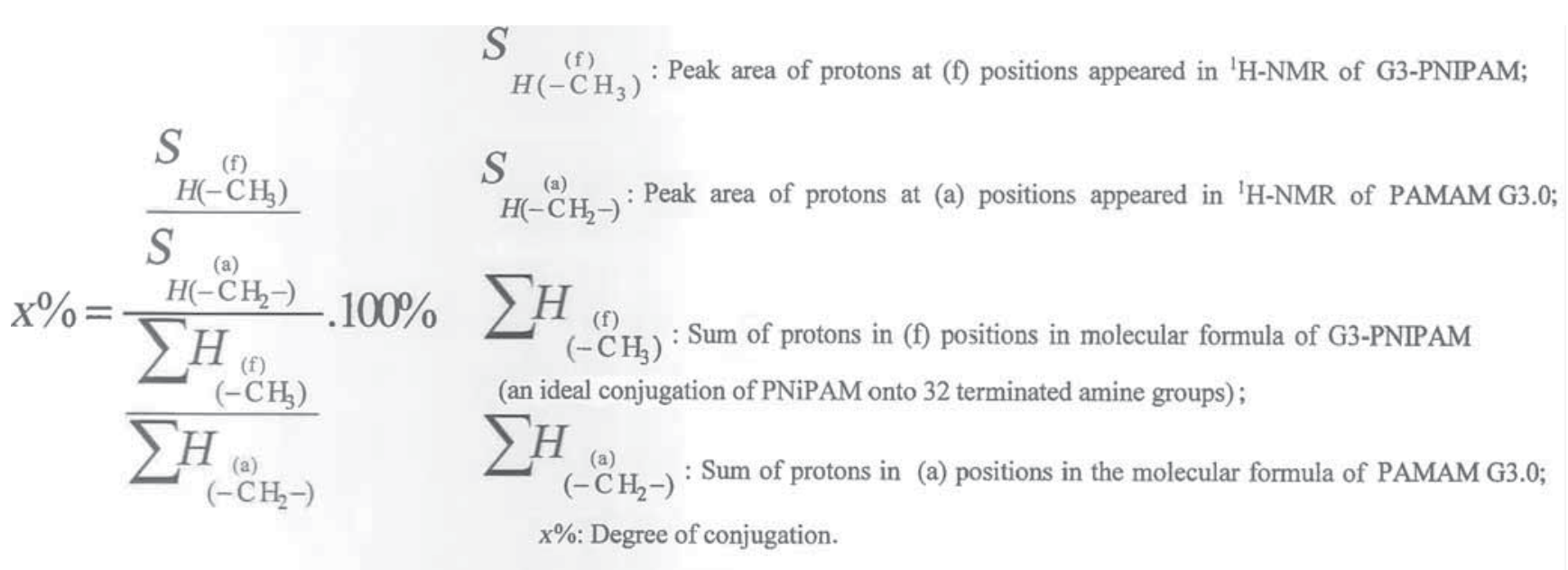

Formula 1: Method to calculate the degree of conjugation.

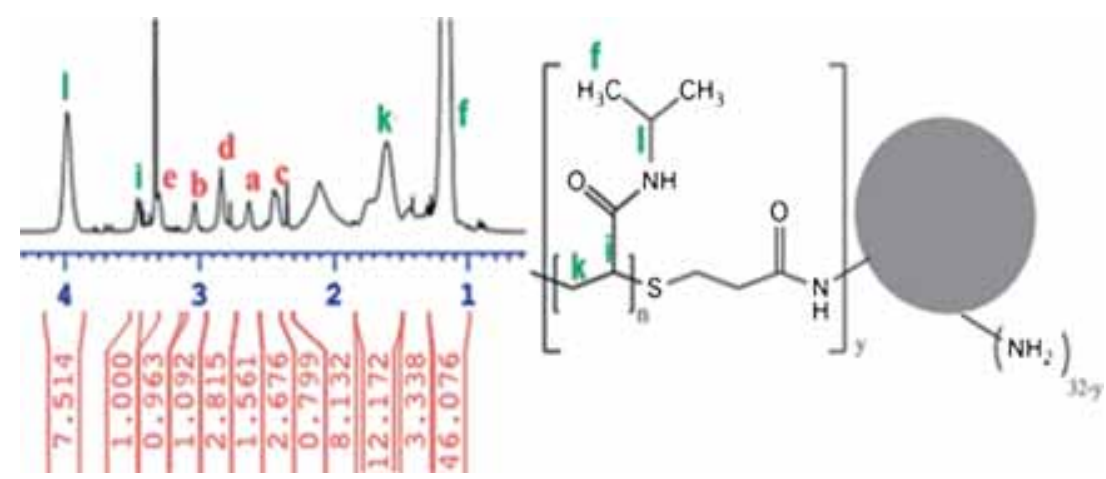

Figure 4. ${ }^{1} \mathrm{H}-\mathrm{NMR}$ spectrum of the PAMAM G3.0-PNIPAM.

Table 1. The conversion (\%) and molecular weight (Mw) of the PAMAM G3.0-PNIPAM.

\begin{tabular}{|c|c|c|c|c|}
\hline $\begin{array}{l}\text { Group ratio of PAMAM } \\
\text { G3.0 : PNIPAM }\end{array}$ & $\begin{array}{l}\text { Number of conjugated } \\
\text { groups per PAMAM }\end{array}$ & $\begin{array}{c}\text { Molecular weight } \\
\left(\mathrm{g} \mathrm{mol}^{-1}\right) ;{ }^{1} \mathrm{H} \text { NMR }\end{array}$ & $\begin{array}{l}\text { Phase transition } \\
\text { temperature }\left({ }^{\circ} \mathrm{C}\right)\end{array}$ & Sample \\
\hline $1: 5.5$ & 3.4 & 30,700 & 37.5 & G3.0 : PNIPAM $_{3.4}$ \\
\hline $1: 8$ & 5.1 & 42,600 & 34.0 & $\mathrm{G}_{3.0}:$ PNIPAM $_{5.1}$ \\
\hline $1: 10$ & 7.0 & 55,900 & 33.0 & $\mathrm{G}_{3.0}:$ PNIPAM $_{7.0}$ \\
\hline
\end{tabular}




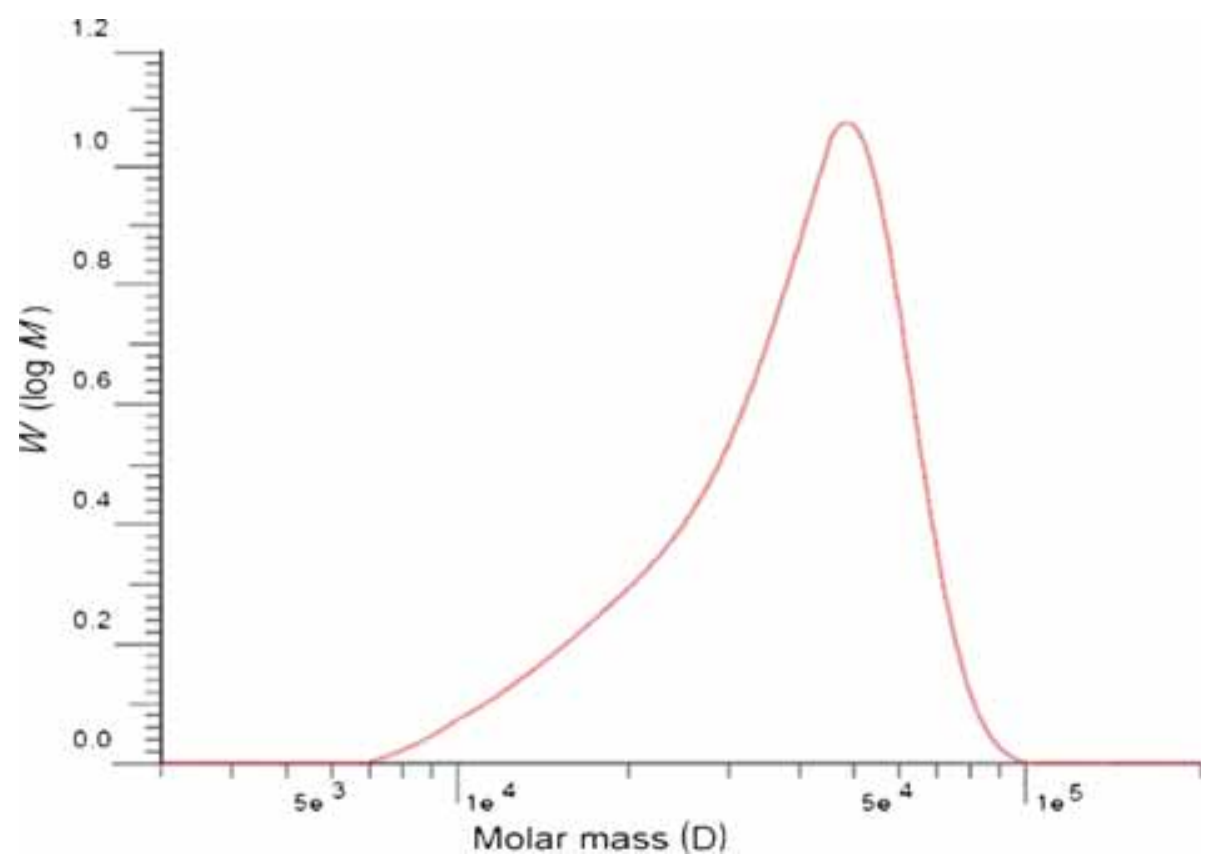

Figure 5. GPC result of the PAMAM G3.0-PNIPAM.
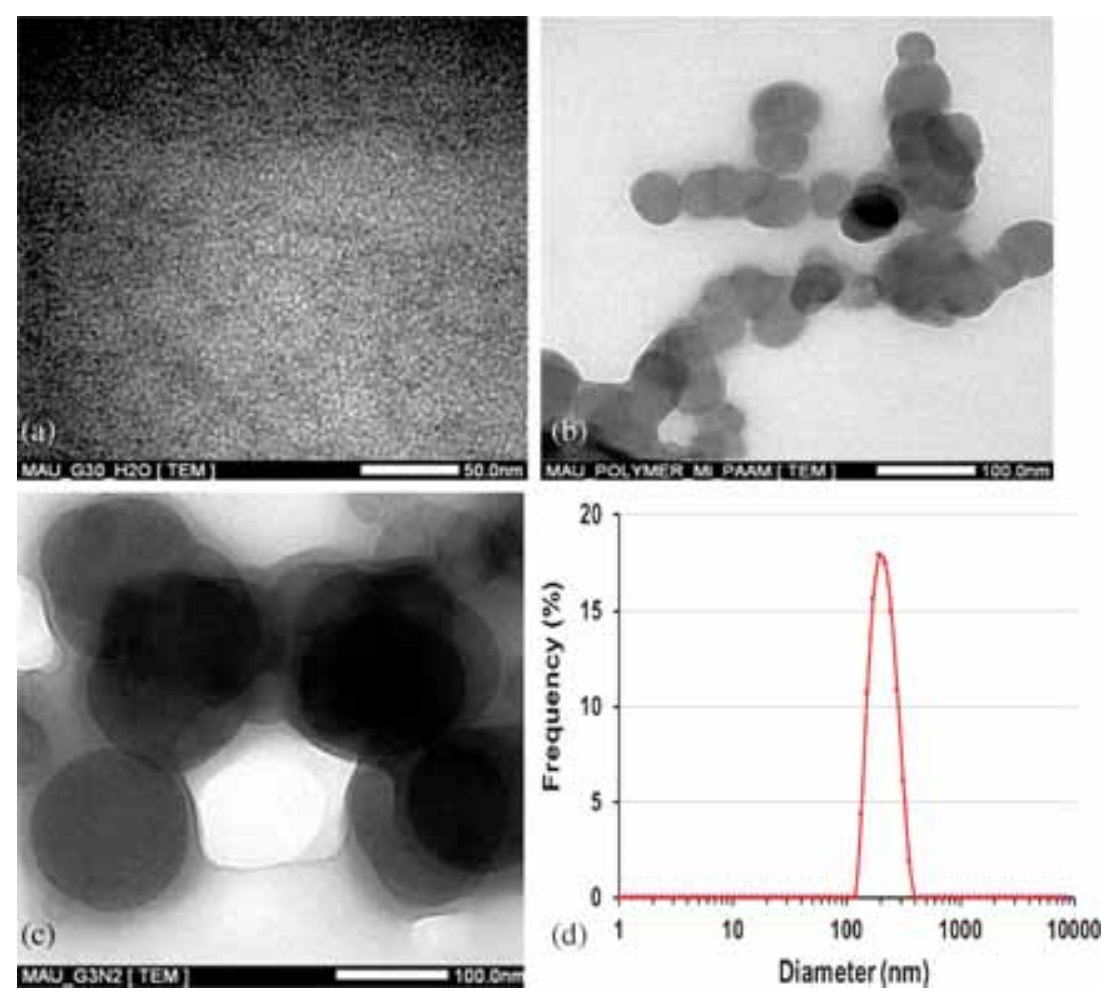

Figure 6. TEM images of (a) PAMAM G3.0, (b) PNIPAM, (c) PAMAM G3.0PNIPAM and (d) size distribution of PAMAM G3.0-PNIPAM with DLS measured at $37^{\circ} \mathrm{C}$.

It could be determined that PAMAM dendrimer provided high degree of surface functionality and versatility, in which the more surface group of PAMAM was modified and the higher efficacy was gained. Yet, to control the appropriate condition of the system with a sustainable size as well as molecular weight, the PNIPAM-group-grafted-PAMAM dendrimer was evaluated for the real size with GPC method and the obtained result was indicated (figure 5) that the 
Mw of PAMAM G3.0-PNIPAM 5.1 was $39,600 \mathrm{~g} \mathrm{~mol}^{-1}$. The value was approximately equal to the $\mathrm{Mw}$ calculated from ${ }^{1} \mathrm{H}-\mathrm{NMR}$ spectra with the same ratio.

Further, the typical characteristics of thermo-sensitive dendritic nanocarrier was determined by the evaluation of the phase transition temperature of the PAMAM G3.0PNIPAM $_{5.1}$, at which transparent solution changed into opaque solution when the copolymer solution increased its temperature. The phenomena of G3-PNIPAM sample was changed sharply from transparency to opacity and its transmission decreased drastically at more than $34^{\circ} \mathrm{C}$ when compared to the transition of PNIPAM at around $31.5^{\circ} \mathrm{C}$. The phase transition temperature of PAMAM G3.0-PNIPAM and PAMAM G3.0-PNIPAM ${ }_{7.0}$ carriers below $37^{\circ} \mathrm{C}$ (which is in blood heat) could be significant potency in drug delivery. Remarkably, PAMAM G3.0-PNIPAM ${ }_{5.1}$ is a potential candidate in this study.

Additionally, the morphology of PAMAM G3.0, PNIPAM and PAMAM G3.0-PNIPAM ${ }_{5.1}$ could be well-defined by TEM at $37^{\circ} \mathrm{C}$ (figure 6) with the size of PAMAM G3.0 and PNIPAM-COOH approximately at 5 and $50 \mathrm{~nm}$ at $37^{\circ} \mathrm{C}$, respectively. After conjugation, the size of PAMAM was clearly recognized in the range of $120-150 \mathrm{~nm}$ with the temperature above $35^{\circ} \mathrm{C}$. An obvious result from PNIPAM chains undergoing hydrophobic interactions since the temperature of copolymer solution is increased. Furthermore, the result was also confirmed by the dynamic light scattering (DLS) measurement which exhibited average size of nanocarriers around $190 \mathrm{~nm}$ (figure 6d). The size of the PAMAM G3.0-PNIPAM 5.1 was measured by DLS indicated larger particle size than that by TEM. This was explained that DLS measurement was done in colloidal solution, while TEM analysis was observed in dried samples of the nanocarriers with a partial contraction of its structure. In other words, the size of particles under DLS measurement which was affected by Brownian motion gave mean hydro-dynamic size usually larger than the particles' size measured by TEM method as it included a few solvent layers [23]. Hence, it was strongly confirmed that PAMAM G3.0 dendrimer was functionalized with PNIPAM-COOH.

\subsection{Drug loading and releasing abilities}

To evaluate the drug-loading efficacy, HPLC technique was used to estimate the amount of free 5-FU drug which could not be entrapped in G3.0-PNIPAM 5.1 nanocarriers at $37^{\circ} \mathrm{C}$. 5 -FU is prepared separately with six different concentrations $\left(\mathrm{mg} \mathrm{ml}^{-1}\right): 0.00125 ; 0.0025 ; 0.005 ; 0.01 ; 0.02$, then based on the HPLC measurements, the standard curve equation is constructed with different measurements of originally loading 5-FU drug at $20 \mathrm{mg}$. Following the standard curve chart, the amount of drug in the PAMAM G3.0-PNIPAM ${ }_{5.1}$ was calculated in comparison with PAMAM G3.0 dendrimer. The nanocarrier exhibited a higher drug loading $(\mathrm{DL}=7.79 \%)$ and entrapment efficiency ( $\mathrm{EE}=42.25 \%$ ) of 5 -FU as compared to PAMAM dendrimer (with $\mathrm{DL}=2.25 \%$ and $\mathrm{EE}=$ $11.52 \%)$. A significant increment in drug-loading capacity of the PAMAM G3.0-PNIPAM ${ }_{5.1}$ nanocarrier compared with the original PAMAM G3.0 dendrimer could be explained that many hydrophobic 5-FU molecules were entrapped in the inner cavity space of dendrimer and hydrophobic domain formed from hydrophobic interaction of PNIPAM resulting in increasing amount of loaded drug. The loading and entrapment efficiency is shown in table 1.

The free 5-FU was initial burst release with the amount accounting for $90 \%$ of loaded 5-FU from dialysis membrane, whereas thermo-sensitive PAMAM G3.0-PNIPAM ${ }_{5.1}$ nanocarrier could maintain merely $96 \%$ of 5 -FU in its nanostructure at the same release time (figure 7). After $5 \mathrm{~h}$, the amount of free 5-FU was released cumulatively from the membrane completely (approximately 98\%), while there was around $30 \%$ and $10 \%$ of 5-FU released from PAMAM G3.0 and PAMAM G3.0-PNIPAM ${ }_{5.1}$ nanocarriers, respectively. The cumulative release of 5-FU reached $37 \%$ after $114 \mathrm{~h}$. Whilst, 5-FU encapsulated in PAMAM G3.0-PNIPAM ${ }_{5.1}$ was at initial burst release in the first $24 \mathrm{~h}$ with accumulative

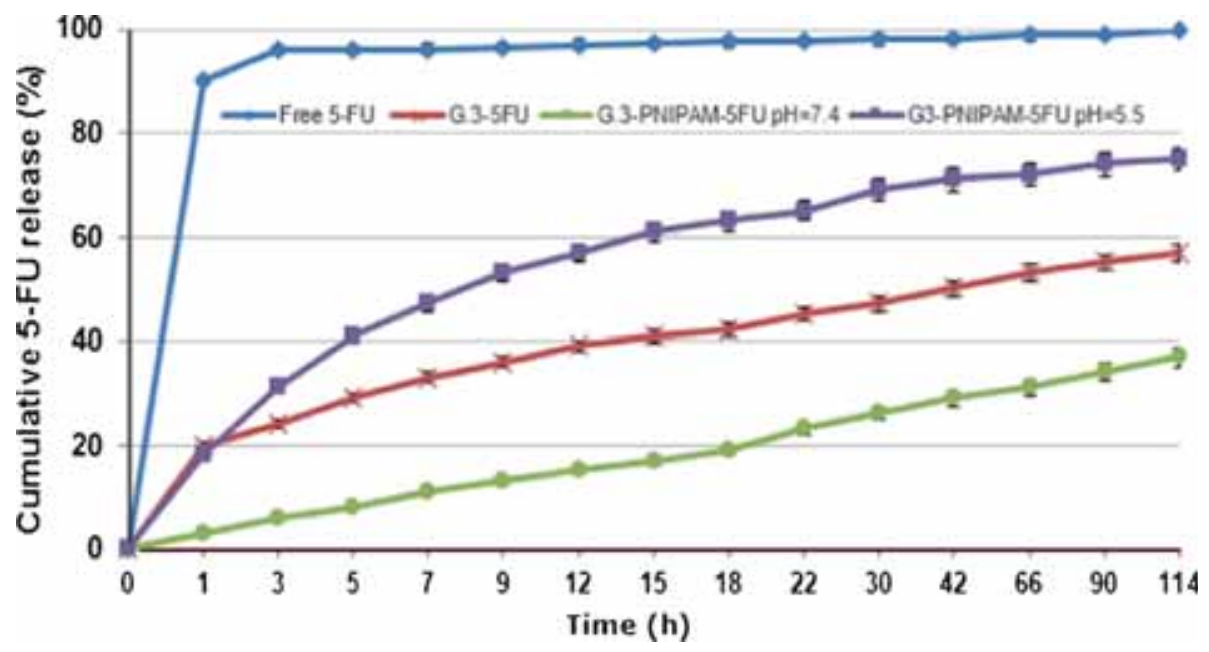

Figure 7. Release profile of free-loaded 5-flourouracil (5-FU) and loaded nanocarriers at $\mathrm{pH} 5.5$ and $7.4, t=37^{\circ} \mathrm{C}$. 


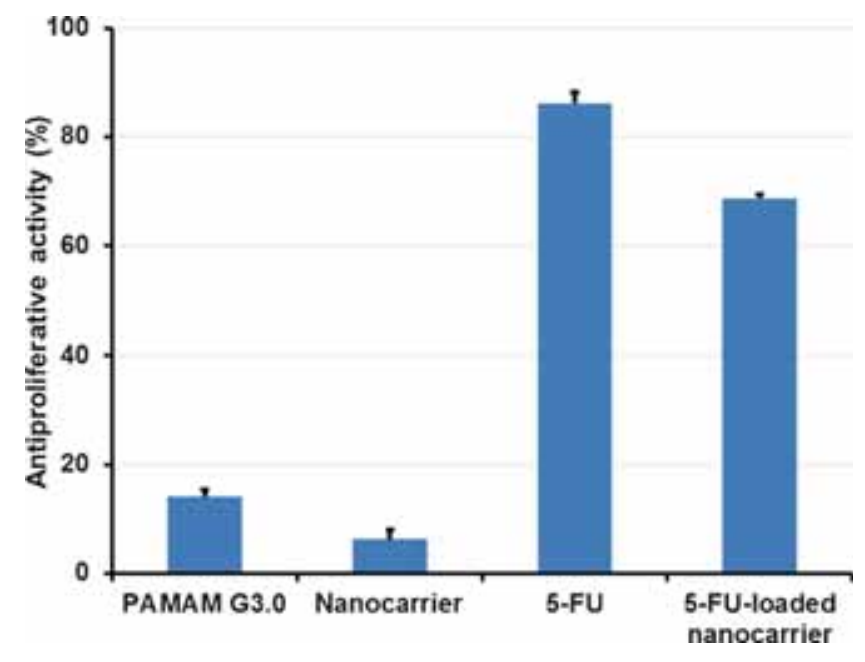

Figure 8. Anti-proliferative activity at screening concentration $\left(100 \mu \mathrm{g} \mathrm{ml}^{-1}\right)$ of PAMAM G3.0, PAMAM G3.0-PNIPAM nanocarrier, 5-FU and 5-FU-loaded nanocarrier.

release rate of approximately $60 \%$, then with a steady increase to $75 \%$ until $114 \mathrm{~h}$. The result is reasonable because strong interaction of 5-FU with hydrophobic PNIPAM domain and the drug encapsulation in the inner cavity space of the NIPAM layer-coated dendrimer contributed to control the drug sustainably from the nanocarrier in the plasma.

Interestingly, an acidic media also impacted on the behaviour of 5-FU release that indicated in figure 7. 5-FUloaded PAMAM G3.0-PNIPAM 5.1 nanocarrier performed a higher release rate of the drug at $\mathrm{pH} 5.5$ as compared at $\mathrm{pH}$ 7.4. This could be explained that the acidic media contributed to protonation and increment in swelling degree of the nanocarriers resulting in a higher leaked drug from the platform [24]. This may be significant to reduce side-effects of the drug in the blood plasma and appropriate to control the drug release in the tumour site.

\subsection{Cytotoxicity assay}

MCF-7 breast cancer cell was used to evaluate cytotoxic behaviour of PAMAM G3.0, thermo-sensitive nanogel PAMAM G3.0-PNIPAM, free 5-FU and 5-FU loaded PAMAM G3.0PNIPAM using sulforhodamine B dye molecule. The result indicated that PAMAM G3.0 and its thermo-sensitive derivative were relatively non-cytotoxic at the experimental condition. At the same concentration, the drug-encapsulated nanogel exhibited a high anti-proliferative activity on the cancer cells whilst free 5-FU performed a high toxicity against the cancer cell growth (figure 8).

The result could indicate that 5-FU encapsulated PAMAM G3.0-PNIPAM was less cytotoxic than free 5-FU which had a high cytotoxicity. Our previous study also found that 5-FU shows high toxicity with MCF-7 with a 50\% inhibition concetration $\left(\mathrm{IC}_{50}\right)$ value at $1.625 \pm 0.419 \mu \mathrm{g} \mathrm{ml}{ }^{-1}$ [10]. With high drug loading efficiency $(\mathrm{DL})=7.79 \%$ and the slowly released drug from the nanocarrier to inhibit $68.27 \pm 0.75 \%$ cell growth. The thermo-sensitive nanocarrier PAMAM G3.0-PNIPAM could be expected to reduce sideeffects of 5-FU as well as studied further to apply as an effective anticancer drug delivery.

\section{Conclusion}

Thermo-sensitive nanocarriers were successful in synthesis and characterization of drug delivery. The obtained results indicated that the nanocarrier increased drug-loading efficiency and controlled drug release. The drug-encapsulated nanogel exhibited a high anti-proliferative activity on the MCF-7 cancer cells. These outstanding achievements might take a great contribution to further studies and development of dendrimer-based nanocarrier applications.

\section{Acknowledgements}

This work was supported by Vietnam National Foundation for Science and Technology Development (NAFOSTED) under grant number 106-YS.99-2013.29. We are grateful to Mr Lian Hock Chuan from Horiba Instruments, Singapore Pte Ltd, for kindly measuring size distribution of PAMAM G3.0-PNIPAM nanocarrier.

\section{References}

[1] Kazunori K, Kwon G S, Masayuki Y, Teruo O and Yasuhisa S 1993 J. Control. Release 24119

[2] Putnam D and Kopecek J 1995 Adv. Polym. Sci. 12255

[3] Greco F and Vicent M J 2009 Adv. Drug Deliv. Rev. 61 1203

[4] Tomalia D A, Baker H, Dewald J, Hall M, Kallos G, Martin S et al 1985 Polym. J. 17117

[5] Nguyen H, Nguyen C K, Nguyen N H and Tran N Q 2014 J. Nanosci. Nanotechnol. 164106

[6] Sönke S and Tomalia D A 2005 Adv. Drug. Deliv. Rev. 57 2106

[7] Wolinsky J B and Grinstaff M W 2008 Adv. Drug. Deliv. Rev. 601037

[8] Bharathi D, Anja J, Srinivasulu C, Shibu T, Girish S, Daniel O et al 2007 J. Biomed. Nanotechnol. 3384

[9] Bhadra D, Bhadra S, Jain S and Jain N K 2003 Int. J. Pharm. 257111

[10] Ly T U, Tran N Q, Hoang T K D, Phan K N, Truong H N and Nguyen C K 2013 J. Biomed. Nanotechnol. 9213

[11] Kukowska-Latallo J F, Candido K A, Cao Z, Nigavekar S S, Majoros I L J, Thomas T P et al 2005 Cancer Res. 65 5317

[12] Virendra G, Vijayaraj G, Rakesh K T and Jain N K 2007 Curr. Pharm. Des. 13415

[13] Bai S and Ahsan F 2009 Pharm. Res. 26539 
[14] Chen J P, Leu Y L, Fang C L, Chen C H and Fang J Y 2011 Int. J. Pharm. 100655

[15] Simona M, Julien N and Patrick C 2013 Nat. Mater. 12 991

[16] Ward M A and Georgiou T K 2011 Polymer 31215

[17] Shen Z Y, Ma G H, Dobashi T, Maki Y and Su Z G 2008 Eur. J. Pharm. Sci. 35271

[18] Jansson J, Schille K, Olofsson G, Cardoso R and Loh W 2004 J. Phys. Chem. B 10882

[19] Castro E, Mosquera V and Katime I 2012 Nanomater. Nanotechnol. 21
[20] Nguyen T B T, Nguyen T T C, Tran H C, Nguyen C K and Tran N Q 2015 Int. J. Polym. Anal. Charact. 20 57

[21] Papazisis K T, Geromichalos G D, Dimitriadis K A and Kortsaris A H 1997 J. Immunol. Methods 208 151

[22] Vichai V and Kirtikara K 2006 Nat. Protoc. 11112

[23] Lee D H, Cho G S, Lim H M, Kim D S, Kim C Y and Lee S H 2013 J. Ceram. Process. Res. 14274

[24] Fuciños C, Fuciños P, Míguez M, Katime I, Pastrana L M and Rúa M L 2014 PLoS One 9 e87190 